\title{
BMJ Open Advance care planning uptake among patients with severe lung disease: a randomised patient preference trial of a nurse-led, facilitated advance care planning intervention
}

\author{
Craig Sinclair, ${ }^{1}$ Kirsten Anne Auret, ${ }^{1}$ Sharon Frances Evans, ${ }^{2}$ Fiona Williamson, ${ }^{1}$ \\ Siobhan Dormer, ${ }^{3}$ Anne Wilkinson, ${ }^{4}$ Kim Greeve, ${ }^{5}$ Audrey Koay, ${ }^{5}$ Dot Price, ${ }^{6}$ \\ Fraser Brims ${ }^{3}$
}

To cite: Sinclair C, Auret KA, Evans SF, et al. Advance care planning uptake among patients with severe lung disease: a randomised patient preference trial of a nurseled, facilitated advance care planning intervention. BMJ Open 2017;7:e013415. doi:10.1136/bmjopen-2016013415

- Prepublication history and additional material is available. To view please visit the journal (http://dx.doi.org/ 10.1136/bmjopen-2016013415).

Received 11 July 2016 Revised 7 December 2016 Accepted 26 January 2017

CrossMark

For numbered affiliations see end of article.

Correspondence to Dr Craig Sinclair; craig.sinclair@rcswa.edu.au

\section{ABSTRACT}

Objective: Advance care planning (ACP) clarifies goals for future care if a patient becomes unable to communicate their own preferences. However, ACP uptake is low, with discussions often occurring late. This study assessed whether a systematic nurse-led ACP intervention increases ACP in patients with advanced respiratory disease.

Design: A multicentre open-label randomised controlled trial with preference arm.

Setting: Metropolitan teaching hospital and a rural healthcare network.

Participants: 149 participants with respiratory malignancy, chronic obstructive pulmonary disease or interstitial lung disease.

Intervention: Nurse facilitators offered facilitated ACP discussions, prompted further discussions with doctors and loved ones, and assisted participants to appoint a substitute medical decision-maker (SDM) and complete an advance directive (AD).

Outcome measures: The primary measure was formal (AD or SDM) or informal (discussion with doctor) ACP uptake assessed by self-report (6 months) and medical notes audit. Secondary measures were the factors predicting baseline readiness to undertake ACP, and factors predicting postintervention ACP uptake in the intervention arm.

Results: At 6 months, formal ACP uptake was significantly higher $(p<0.001)$ in the intervention arm $(54 / 106,51 \%)$, compared with usual care $(6 / 43,14 \%)$. ACP discussions with doctors were also significantly higher $(p<0.005)$ in the intervention arm $(76 / 106$, $72 \%)$ compared with usual care $(20 / 43,47 \%)$. Those with a strong preference for the intervention were more likely to complete formal ACP documents than those randomly allocated. Increased symptom burden and preference for the intervention predicted later ACP uptake. Social support was positively associated with ACP discussion with loved ones, but negatively associated with discussion with doctors.

Conclusions: Nurse-led facilitated ACP is acceptable to patients with advanced respiratory disease and effective in increasing ACP discussions and completion

\section{Strengths and limitations of this study}

- This randomised controlled trial includes a patient preference arm, to more closely match the real-world clinical environment.

- Longitudinal follow-up enables assessment of multiple aspects of advance care planning uptake among a specific patient cohort at high risk of mortality.

- A higher than expected number of patients who strongly preferred to receive the intervention means the study arms are unbalanced.

- Patient attrition across the follow-up period and self-selection associated with the use of a preference arm complicate the interpretation of study data.

- A detailed description of characteristics associated with the facilitated advance care planning intervention provides implications for practitioners.

of formal documents. Awareness of symptom burden, readiness to engage in ACP and relevant psychosocial factors may facilitate effective tailoring of ACP interventions and achieve greater uptake.

Trial registration number: ACTRN12614000255684.

\section{BACKGROUND}

Advance care planning (ACP) is an ongoing process of discussion between patients, family, carers and health professionals aimed at clarifying goals for future care and facilitating decision-making in situations when a patient is unable to make decisions or communicate their own preferences. ${ }^{1}$ While ACP has traditionally been understood and measured in terms of the completion of a formal advance directive (AD) to specify preferences for medical treatments or nomination of a 
substitute healthcare decision-maker (SDM), there is growing awareness that such approaches do not capture the full breadth of the planning and discussion involved. $^{23}$

Recent systematic reviews have supported the efficacy of ACP interventions, ${ }^{4}{ }^{5}$ particularly those including facilitated communication approaches in addition to written directives. ${ }^{4}$ ACP is associated with positive outcomes in end-of-life care including reduced psychological morbidity among bereaved family members, lower likelihood of dying in hospital and higher likelihood of planned hospice admission. ${ }^{36}$

Patients with severe respiratory disease, such as lung cancer or chronic obstructive pulmonary disease (COPD), have been identified as a group for whom ACP is particularly relevant, ${ }^{7-9}$ as they experience heavy symptom burden with a marked impact on quality of life and care needs. ${ }^{10}{ }^{11}$ Prognosis among these patients can be poor; the 1-year mortality rate in metastatic non-small cell lung cancer is over $70 \%{ }^{12}$ and is over $20 \%$ among patients hospitalised for acute exacerbation of COPD. ${ }^{13}$ While malignant respiratory disease has a somewhat predictable illness trajectory, COPD is characterised by significant fluctuation, in which periods of stability or gradual decline are interspersed with acute exacerbations, any of which may be fatal. ${ }^{11}$ A study of patients with advanced lung cancer and end-stage COPD showed that while both groups expressed strong preferences for comfort-focused care, those with COPD were significantly more likely to receive invasive therapies. ${ }^{14}$

Despite this identification of potential benefit, ACP rates remain low among patients with severe respiratory disease. ${ }^{15}{ }^{16}$ Patient-related barriers include a lack of information about their condition, ${ }^{17}{ }^{18}$ a belief that clinicians will initiate ACP discussion 'when the time is right', ${ }^{19}$ and in some cases, a preference to avoid discussion about ACP and end-of-life care. ${ }^{8}{ }^{20}$ Patients with COPD in particular are often unclear about their prognosis, unaware of their likely illness trajectory and not informed about the types of healthcare decisions they may face in the future. ${ }^{15} 1718$ Doctors also report a range of barriers to ACP discussion including lack of time, ${ }^{21}$ concern about upsetting patients ${ }^{22}$ and prognostic uncertainty. ${ }^{23}$ Previous research has established the relevance and effectiveness of nurses in ACP facilitation, in community and hospital settings. ${ }^{6} 24$

Uptake of ACP can be usefully conceptualised as a process, with modified stage-based models of health behaviour change which describe patient 'readiness' (ie, precontemplation, contemplation, preparation, action/ maintenance) to engage in the various aspects of ACP including discussions with loved ones, discussions with doctors and completion of formal documents. ${ }^{25} 26$ Validated interview and survey tools enable nuanced longitudinal measurement of psychological stages of 'readiness' over time, as opposed to measures focusing solely on 'completion'. 2728 This construct of 'readiness' to undertake ACP has been shown to be associated with illness-related experiences, ${ }^{8}{ }^{29}$ experiences with end-of-life care among others, ${ }^{29}{ }^{30}$ social support ${ }^{624}$ and proactive intervention by clinicians. ${ }^{26}$

The primary research question addressed in this paper is whether a systematic nurse-led, facilitated ACP intervention is effective in increasing ACP readiness and uptake among patients with advanced respiratory disease. The secondary aims are to (1) identify patient factors associated with ACP readiness at baseline, and (2) identify patient and contextual factors associated with ACP uptake among those who were assigned to receive the facilitated ACP intervention.

\section{METHODS}

\section{Study design}

This study was a multicentre open-label randomised controlled trial of nurse-led facilitated ACP with a preference $\mathrm{arm}^{31}$ and a 2:1 randomisation protocol in favour of the intervention. The preference arm enabled participants with strong preferences (to either receive or avoid the intervention) to be assigned to their preferred group.

\section{Study setting}

The study was implemented in a metropolitan and a rural setting in Western Australia (WA). The metropolitan setting was a tertiary hospital respiratory department. The rural setting, $\sim 400 \mathrm{~km}$ away, consisted of general practice (GP) clinics, residential aged care facilities (RACF) and the local regional hospital in a town of $\sim 30000$ people.

\section{Participant eligibility criteria}

Patients were eligible for the study if they were diagnosed with a chronic, severe respiratory disease (lung cancer, mesothelioma, malignant pleural effusion, COPD or interstitial lung disease); fulfilled one or more of the general or disease-specific criteria predicting 'high risk' of death, based on the Gold Standards Framework ${ }^{32}$ (table 1); were receiving treatment in one of the study settings; and were over 18 years of age. Patients were excluded if they lacked capacity to consent; did not speak English; had previously completed a formal AD (but not excluding those who had previously nominated an SDM); were on an 'end-of-life' pathway or otherwise expected to die in the next 48 hours.

\section{Recruitment and randomisation}

In the metropolitan setting, potential participants were identified through department database searches, clinic letter reviews and direct referrals by ward staff. In the rural setting, potential participants were identified through GP database searches, direct referrals from participating GP clinics, regular meetings with RACF coordinators and attendance at clinical meetings in the regional hospital. The nurse ACP facilitator confirmed the eligibility of each patient with their primary treating doctor, and sought the doctor's permission prior to approaching them and explaining the study. 
Table 1 High-risk criteria for patient inclusion. Eligible patients were diagnosed with an eligible respiratory disease, and met one or more of any of the general indicators or disease-specific triggers, or a 'no' in answer to the 'surprise question'

\section{General indicators:}

1. WHO/ECOG performance status of 3 or greater ${ }^{33}$

2. Unstable and/or deteriorating symptom burden

3. Decreasing response to treatments

4. Weight loss $>10 \%$ in past 6 months

5. Serum albumin $<25 \mathrm{~g} / \mathrm{L}$

6. Repeated unplanned hospital admission(s) for a respiratory symptom

\section{Disease-specific triggers}

COPD:

1. Severe disease on spirometry $\left(F E V_{1}\right.$ $<25 \%$ predicted)

2. Recurrent hospital admissions (3 or more in a 12-month period)

3. Fulfils LTOT criteria

4. MRC grade shortness of breath $4-5$

5. Right heart failure

6. 6 weeks or more of systemic steroids in past 6 months

7. Respiratory failure within the past 12 months requiring intensive care unit admission or non-invasive ventilation

\section{The surprise question:}

Ask the primary treating doctor responsible for the patient's care: 'Would you be surprised if the patient were to die in the next few months, weeks or days? ${ }^{34}$

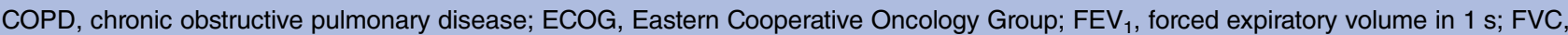
forced vital capacity; LTOT, long-term oxygen therapy; MRC, Medical Research Council.

During the recruitment process, potential participants were informed that as part of participation they may be invited to discuss the type of medical care they would want if they were unable to make decisions and/or communicate their wishes, including discussion about life-sustaining treatments and end-of-life care. Those who consented to the study and expressed a strong preference to receive, or avoid, the facilitated ACP intervention were assigned to their preferred intervention or usual care (control) arm, respectively (called 'Pref-ACP' and 'Pref-CON').

Patients willing to be randomised were allocated (called 'Rand-ACP' and 'Rand-CON') following consent (see figure 1). SFE generated the random allocation sequence in permuted blocks ( $\mathrm{N}=21$ per block), and filled opaque, sealed envelopes with allocation slips. The nurse facilitators asked each patient to select an envelope; both the nurse and patient were blinded to the contents of the envelope prior to allocation. The allocation protocol initially employed a 2:1 randomisation schedule. Following planned preliminary analysis and observation of a higher than expected number of patients expressing a strong preference for the intervention, this was revised to a 1:1 schedule (ethics committee approval 12/2/2015), and recruitment was ended on $16 / 9 / 2015$ due to reporting deadlines.

A target sample size of $n=150$ in each study setting was based on a power calculation of 0.80 , assuming a log-normal distribution, and a $75 \%$ ratio of geometric mean levels of ACP uptake between the usual care and intervention arms.

\section{Intervention: nurse-led facilitated ACP}

The intervention provided nurse-led support to the participant, their family and their doctors to facilitate engagement in ACP. A nurse facilitator was employed in each setting to coordinate recruitment, deliver the intervention and collect surveys. Both were senior nurses with extensive experience in communication with severely ill patients. Intervention fidelity was maintained across the study settings by research team participation in a full-day workshop delivered by an external consultant (using evidence-based resources adapted with permission from Respecting Patient Choices), a detailed study protocol and regular meetings between the nurse facilitators and the broader research team.

Participants assigned to the intervention were offered an appointment with the nurse facilitator to discuss their illness and prognosis, reflect on goals and values for future medical care, talk about these with loved ones and doctors, appoint an SDM, and/or formally document future treatment preferences in an AD.

In the metropolitan setting, the intervention typically occurred in an outpatient clinic, and in the rural setting this occurred in a GP room or in the participant's 


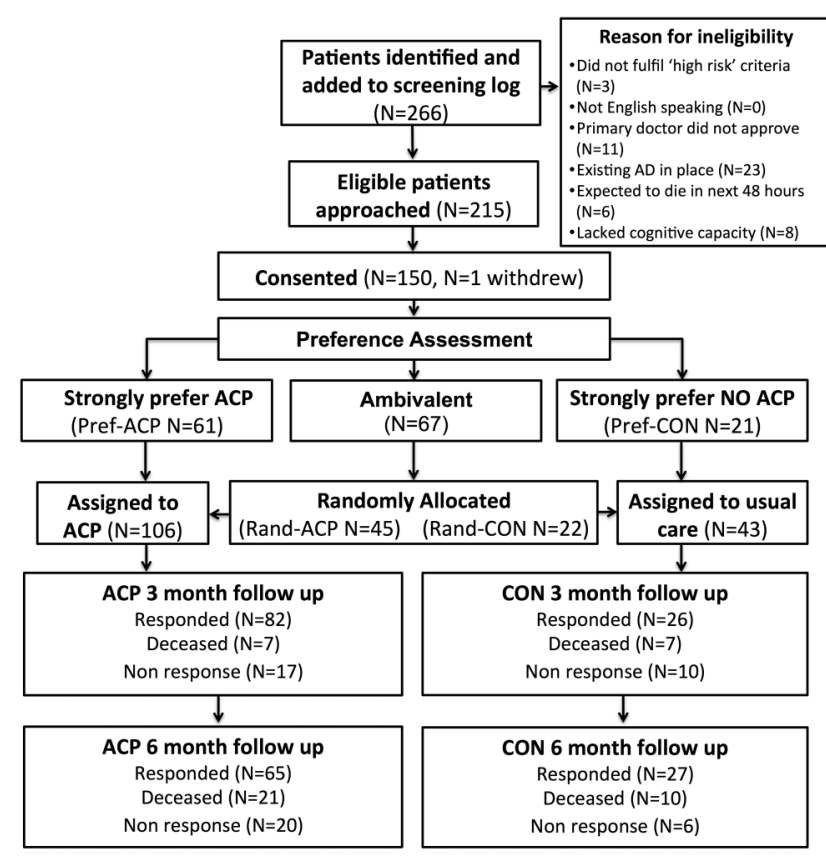

Figure 1 Schematic diagram of participants approached, consented and recruited to trial. ACP, advance care planning; LCP, Liverpool Care Pathway.

home. Follow-up meetings with the nurse facilitator were scheduled opportunistically, or by participant request. The date, length (minutes), location, people present and domains covered in each ACP discussion were recorded on a checklist. Any formal ACP documentation completed as part of the intervention was distributed to the participant, their family carer/s, regular GP and medical records department of the relevant hospital.

\section{Measures}

Participant age, gender, country of birth, religion and highest level of education attained were recorded at baseline. Each participant's primary respiratory diagnosis, secondary respiratory diagnoses (where applicable), Medical Research Council (MRC) breathlessness scale grading and eligibility for long-term oxygen therapy (LTOT) were obtained from medical notes during screening for eligibility. ${ }^{35}$

\section{Primary outcome: ACP uptake}

Two approaches were taken to measuring ACP uptake. First, participant interviews, administered at baseline, 3 and 6 months postconsent, employed a validated survey tool to assess stage of readiness to engage in relevant aspects of ACP: (1) completion of a written AD, (2) documentation of an SDM, (3) discussion about lifesustaining treatments with loved ones and (4) discussion about life-sustaining treatments with doctors. ${ }^{27}$ Second, at 12 months after consent, time of death or study end point (whichever occurred first), participants' medical notes were audited from the time of consent to assess the presence of 'formal' or 'informal' documentation of patient preferences regarding future medical care.
Inclusion of the notes audit meant that ACP uptake could be assessed for patients who had died or were lost to follow-up prior to the 6-month follow-up surveys. Medical notes audit was undertaken by the nurse facilitators using a structured audit tool. ${ }^{36}$ The sections of the notes containing evidence of ACP uptake were scanned and de-identified, to allow inspection by the analysts in the research team (CS and SFE), who ensured that documentation met the definition of ACP uptake.

For the primary outcome, 'formal ACP uptake' was defined as self-reported completion of a written $\mathrm{AD}$ (in WA, this means an 'Advance Health Directive' ${ }^{\text {'37 }}$ or a 'My Advance Care Plan' form ${ }^{38}$ ), or written nomination of an SDM (an 'Enduring Power of Guardianship' ${ }^{39}$ ) at 6-month follow-up. For those who died or were lost to follow-up prior to the 6-month survey, the self-report data were supplemented by medical notes audit. 'Informal ACP uptake' was defined as self-reported completion of at least one discussion about life-sustaining treatments with doctors at 6-month follow-up, or documentation of ACP conversations found at notes audit.

\section{Secondary measures}

Participant self-reported health-related quality of life (HRQOL), satisfaction with healthcare and social support was measured at baseline. The EuroQol 5 Dimensions 5 Level Survey (EQ-5D-5L) scores HRQOL on five dimensions (mobility, ability to self-care, ability to undertake usual activities, pain and anxiety/depression) and a global HRQOL visual analogue scale (VAS) scored continuously from 1 to 100 . Index values for the EQ-5D-5L were calculated with algorithms derived from the 'cross-walk' mapping to existing EQ-5D-3L data sets, using UK population data (in the absence of Australian validation data). ${ }^{40}$

Satisfaction with healthcare was assessed using the Patient Satisfaction Index (PSI), a validated 23-item instrument, summated to give a global score reflecting satisfaction with level of healthcare received, involvement in healthcare decision-making and interactions with healthcare professionals over the previous 3 months. ${ }^{41}$ Social support was measured by summating the seven-item ENRICHD tool, which assesses instrumental and emotional aspects of support, rather than the size of the social network. ${ }^{42}$ Owing to the small amount of missing data (0.7\% for ENRICHD, 1.7\% for PSI) and unidimensional scale characteristics, missing data points were imputed from each participant's mean score. ${ }^{43}$

\section{Data analysis}

Variables collected at baseline were assessed for matching across trial arms, with $\chi^{2}$ tests for categorical variables and one-way analysis of variance for continuous variables. The primary outcome was assessed by Fisher's exact test and relative risk estimation of ACP uptake with assignment (facilitated ACP vs usual care), preference (strong preference for ACP vs ambivalent) and time (baseline vs 6-month follow-up). 
Secondary exploratory analyses determined the factors predicting 'baseline ACP readiness' among all patients, and 'postintervention formal ACP uptake' and 'postintervention informal ACP uptake', among patients assigned to receive the facilitated ACP intervention. Significant predictors were assessed with separate multivariate logistic regression models, using type III sums of squares estimation, with forced entry and stepwise, backwards elimination of non-significant variables $(p>0.2)$. Initial models included demographic variables (gender, age, country of birth, level of education), baseline survey scores (EQ-5D-5L index scores and global VAS, satisfaction with healthcare, social support) and clinical variables (malignant disease, COPD, MRC breathlessness scale and LTOT eligibility). In addition, analysis of 'postintervention formal ACP uptake' and 'postintervention informal ACP uptake' included baseline ACP readiness, assigned group, hospital admission during the follow-up period, number of ACP discussions undertaken with the nurse facilitator and presence of a family member or carer in an ACP discussion as potential predictors.

The study was registered with the Australia and New Zealand Clinical Trials Registry (ACTRN12614000255684, Registration date: 10/3/2014).

\section{RESULTS}

Over a 15-month period, 266 patients were screened as being potentially eligible across both sites, of whom 215 were confirmed eligible and invited to participate. Of these participants, 150 consented, with one withdrawing before assignment. Eighty-two patients had a strong preference for a particular study arm (ACP or usual care) while 67 were ambivalent and hence randomly allocated to either the intervention or usual care (see figure 1). Owing to the lower than expected recruitment rates, data were combined across sites to maintain adequate study power.

Demographics and clinical profile at baseline are presented in table 2 . The sample was predominantly male $(94 / 149,63 \%)$, with a moderate-to-low educational status. COPD was the most common respiratory diagnosis $(95 / 149,64 \%)$ and $54(36 \%)$ participants were deceased at 12-month follow-up. Baseline rates of ACP discussion with loved ones or doctors did not vary significantly across the different diagnosis groups.

Among those who were randomly allocated, there were no significant differences in primary respiratory diagnoses or measures of disease severity, and most measures of baseline ACP readiness showed no significant differences across study arms. The exception was that those in the Rand-CON group reported higher baseline rates of discussion with their doctor about life-sustaining treatments $(9 / 22,41 \%)$ compared with those in the Rand-ACP group $(8 / 45,18 \%, \mathrm{p}<0.05)$.

Compared with those in the Rand-ACP group, participants in the Pref-ACP group had a lower HRQOL index ( 0.43 vs $0.55, p<0.05)$, lower rates of living with a married or de facto partner (28/61, $46 \%$ in Pref-ACP vs 33/45, $73 \%$ in Rand-ACP, $\mathrm{p}<0.01$ ), higher rates of completing secondary school $(27 / 61,44 \%$ in Pref-ACP vs $8 / 45,18 \%$ in Rand-ACP, $\mathrm{p}<0.01$ ), greater numbers reporting limitations in their ability to self-care $(34 / 61,56 \%$ in Pref-ACP vs $13 / 45,29 \%$ in Rand-ACP, $\mathrm{p}<0.01$ ), higher rates of anxiety or depression (29/61, 48\% in Pref-ACP vs 10/45, $22 \%$ in Rand-ACP, $\mathrm{p}<0.01$ ) and were more likely to be recruited in the metropolitan setting $(35 / 61,57 \%$ in Pref-ACP vs 13/45, 29\% in Rand-ACP, $\mathrm{p}<0.01$ ).

\section{Facilitated ACP intervention}

The characteristics of the facilitated ACP intervention for the 106 participants assigned to the intervention are presented in table 3. In the metropolitan setting, 31 (64\%) participants had at least one discussion with the nurse facilitator; these were predominantly in outpatient clinics (94\%) and lasted an average of $55 \mathrm{~min}$ $(\mathrm{SD}=25.0)$. In the rural setting, $58(100 \%)$ participants had at least one discussion with the nurse facilitator; these were typically home visits $(60 \%)$ and lasted an average of $25 \mathrm{~min}(\mathrm{SD}=16.6)$, with a higher percentage of rural participants $(69 \%$ vs $4 \%, \mathrm{p}<0.001)$ participating in two or more discussions.

\section{ACP readiness and uptake}

The number of participants with formal or informal ACP uptake at baseline and follow-up is presented in table 4. The number of participants who had contemplated or completed different aspects of ACP at baseline and follow-up ( 3 and 6 months) is presented in online supplementary appendix 1 .

For the primary outcome, formal ACP uptake over time (baseline vs 6-month follow-up) found significant effects of assignment and preference. There was an increased likelihood of having ACP uptake at 6-month follow-up (relative risk (RR) $3.65,95 \%$ CI 1.70 to 7.85 ) among those assigned to receive the intervention (54/106, $50.9 \%)$, compared with those assigned to usual care $(6 / 43,14.0 \%)$. There was also increased ACP uptake at 6-month follow-up (RR 2.58, 95\% CI 1.55 to 4.31 ) among those with a strong preference for the intervention (42/61, 68.9\%) compared with those allocated randomly to receive the intervention $(12 / 45,26.7 \%)$. These results were confirmed with logistic regression analysis allowing an interaction between preference and assignment. Among those assigned to ACP intervention, those with a strong preference (Pref-ACP) had an OR of 6.1 (95\% CI 2.6 to 14.3) of ACP uptake, compared with those allocated randomly (Rand-ACP). There was no difference between those allocated randomly to ACP and those assigned to usual care (OR 1.6, 95\% CI 0.5 to 5.8). Informal ACP uptake was significantly higher at 6-month follow-up (76/106, 71.7\%) compared with baseline (33/ $106,31.1 \%, \mathrm{p}<0.001)$ for those assigned to the intervention, while those assigned to usual care did not show a significant difference in uptake over time $(12 / 43,27.9 \%$ vs $20 / 43,46.5 \%$, NS). 
Table 2 Participant characteristics by assigned group (percentages expressed by column)

\begin{tabular}{|c|c|c|c|c|c|c|}
\hline & \multicolumn{2}{|c|}{ Intervention } & \multicolumn{2}{|l|}{ Usual care } & \multicolumn{2}{|l|}{ Preference } \\
\hline & $\begin{array}{l}\text { Pref-ACP } \\
(\mathrm{N}=61)\end{array}$ & $\begin{array}{l}\text { Rand-ACP } \\
(\mathrm{N}=45)\end{array}$ & $\begin{array}{l}\text { Rand-CON } \\
(\mathrm{N}=22)\end{array}$ & $\begin{array}{l}\text { Pref-CON } \\
(\mathrm{N}=21)\end{array}$ & $\begin{array}{l}\begin{array}{l}\text { Pref } \\
(\mathrm{N}=82)\end{array}\end{array}$ & $\begin{array}{l}\text { No Pref } \\
(\mathrm{N}=67)\end{array}$ \\
\hline \multicolumn{7}{|l|}{ Recruitment site } \\
\hline $\mathrm{N}(\%)$ metropolitan hospital & $35(57 \%)$ & $13(29 \%)$ & $5(23 \%)$ & $14(67 \%)$ & $49(60 \%)$ & $18(27 \%)$ \\
\hline $\mathrm{N}(\%)$ rural site & $26(43 \%)$ & $32(71 \%)$ & $17(77 \%)$ & $7(33 \%)$ & $33(40 \%)$ & $49(73 \%)$ \\
\hline \multicolumn{7}{|l|}{ Demographics } \\
\hline Age (median, IQR) & $73(13)$ & $70(12.5)$ & $77.5(8.2)$ & $80(15.5)$ & $74(14)$ & $71(12)$ \\
\hline $\mathrm{N}(\%)$ female & $26(43 \%)$ & $13(29 \%)$ & $6(27 \%)$ & $10(48 \%)$ & $36(44 \%)$ & $19(28 \%)$ \\
\hline $\mathrm{N}(\%)$ married or de facto & $28(46 \%)$ & $33(73 \%)$ & $16(73 \%)$ & $16(76 \%)$ & $44(54 \%)$ & 49 (73\%) \\
\hline $\mathrm{N}(\%)$ born in Australia & $34(56 \%)$ & $25(56 \%)$ & 17 (77\%) & $12(57 \%)$ & $46(56 \%)$ & $42(63 \%)$ \\
\hline $\mathrm{N}(\%)$ observe religion & $26(43 \%)$ & $19(42 \%)$ & $11(50 \%)$ & $11(52 \%)$ & $37(45 \%)$ & $30(45 \%)$ \\
\hline N (\%) Christian & $23(88 \%)$ & $16(84 \%)$ & $8(73 \%)$ & $11(100 \%)$ & $34(41 \%)$ & $24(36 \%)$ \\
\hline $\mathrm{N}(\%)$ other religion & $3(12 \%)$ & $3(16 \%)$ & $3(27 \%)$ & - & $3(4 \%)$ & $6(9 \%)$ \\
\hline \multicolumn{7}{|l|}{ Education level (completed) } \\
\hline $\mathrm{N}(\%)$ not completed secondary & $34(56 \%)$ & 37 (82\%) & $14(64 \%)$ & $8(38 \%)$ & $42(51 \%)$ & $51(76 \%)$ \\
\hline $\mathrm{N}(\%)$ completed secondary & $27(44 \%)$ & $8(18 \%)$ & $8(36 \%)$ & $13(62 \%)$ & 40 (49\%) & $16(24 \%)$ \\
\hline \multicolumn{7}{|l|}{ Primary respiratory diagnosis } \\
\hline $\mathrm{N}(\%)$ malignant disease ${ }^{*}$ & $17(28 \%)$ & $13(29 \%)$ & $8(36 \%)$ & $3(14 \%)$ & $20(24 \%)$ & $21(31 \%)$ \\
\hline $\mathrm{N}(\%)$ COPD & $40(66 \%)$ & $28(62 \%)$ & $12(54 \%)$ & $15(71 \%)$ & $55(67 \%)$ & $40(60 \%)$ \\
\hline $\mathrm{N}(\%)$ interstitial fibrosis & $4(7 \%)$ & $3(7 \%)$ & $1(4 \%)$ & $3(14 \%)$ & $7(8 \%)$ & $4(6 \%)$ \\
\hline $\mathrm{N}(\%)$ other respiratory & - & $1(2 \%)$ & $1(4 \%)$ & - & - & $2(3 \%)$ \\
\hline \multicolumn{7}{|l|}{ Baseline clinical severity } \\
\hline Eligible for LTOT & $26(43 \%)$ & $11(24 \%)$ & $5(23 \%)$ & $10(48 \%)$ & $36(44 \%)$ & $16(24 \%)$ \\
\hline MRC dyspnoea (grade 4-5) & 33 (54\%) & $19(42 \%)$ & $11(50 \%)$ & $11(52 \%)$ & $44(54 \%)$ & $30(45 \%)$ \\
\hline \multicolumn{7}{|l|}{ Baseline survey measures } \\
\hline Social support (mean, SD) & $27.9(5.8)$ & $28(6.2)$ & $28.4(6.8)$ & $27.9(6.9)$ & $27.9(6.0)$ & $28.3(6.3)$ \\
\hline Care satisfaction (mean, SD) & $117(26.9)$ & $114(31.5)$ & 116 (29.2) & $128(39.8)$ & $120(30.9)$ & 115 (30.6) \\
\hline \multicolumn{7}{|l|}{ Health-related quality of life } \\
\hline $\mathrm{N}(\%)$ mobility symptoms $\dagger$ & $44(72 \%)$ & $26(58 \%)$ & $13(59 \%)$ & $12(57 \%)$ & $56(68 \%)$ & $39(58 \%)$ \\
\hline $\mathrm{N}(\%)$ personal care symptoms $\dagger$ & $34(56 \%)$ & $13(29 \%)$ & $3(14 \%)$ & $5(24 \%)$ & $39(48 \%)$ & $16(24 \%)$ \\
\hline $\mathrm{N}(\%)$ usual activity symptoms $\dagger$ & $48(79 \%)$ & $32(71 \%)$ & $14(64 \%)$ & $14(67 \%)$ & $62(76 \%)$ & $46(69 \%)$ \\
\hline $\mathrm{N}(\%)$ pain and discomfort† & $36(59 \%)$ & $20(44 \%)$ & $12(54 \%)$ & $8(38 \%)$ & $44(54 \%)$ & $32(48 \%)$ \\
\hline $\mathrm{N}(\%)$ anxiety and depressiont & $29(48 \%)$ & $10(22 \%)$ & $7(32 \%)$ & $7(33 \%)$ & $36(44 \%)$ & $17(25 \%)$ \\
\hline EQ-5D-5L index (mean, SD) & $0.43(0.21)$ & $0.55(0.24)$ & $0.54(0.24)$ & $0.56(0.24)$ & $0.46(0.22)$ & $0.54(0.22)$ \\
\hline EQ-5D-5L global VAS (mean, SD) & $57.9(21.5)$ & $64.0(16.0)$ & $63.4(20.0)$ & $58.3(17.9)$ & $58.0(20.5)$ & $63.8(17.3)$ \\
\hline
\end{tabular}

Among participants assigned to receive the ACP intervention (Pref-ACP or Rand-ACP) with baseline and 3-month follow-up data available $(\mathrm{N}=82)$, completion of ACP discussions about life-sustaining treatments with loved ones increased from baseline to 3-month follow-up (62\% vs $77 \%, \mathrm{p}<0.05)$. Among participants assigned to usual care (Pref-CON or Rand-CON) with baseline and 3-month follow-up data available $(\mathrm{N}=26)$, ACP discussions with loved ones showed a trend towards increase over time ( $50 \%$ vs $73 \%, \mathrm{p}=0.06)$.

As shown in figure 2, the rate of self-reported AD completion at 6-month follow-up was higher in the Pref-ACP group $(21 / 32,66 \%)$ compared with the Rand-ACP group $(7 / 33,21 \%$; RR $3.09,95 \%$ CI 1.53 to 6.25 , $\mathrm{p}<0.001)$ or compared with those assigned to either usual care group $(1 / 24,4 \%, \mathrm{p}<0.001)$.

\section{Factors associated with ACP uptake}

Factors associated with completion of different aspects of ACP are presented in table 5. Baseline ACP discussion with loved ones was associated with higher social support $(\mathrm{OR}=1.1,95 \%$ CI 1.04 to 1.65$)$, while baseline ACP discussion with doctors was associated with lower HRQOL (OR=0.14, 95\% CI 0.03 to 0.64 ).

Among those assigned to the intervention arm (ie, Pref-ACP or Rand-ACP), the factors associated with formal ACP uptake postintervention were the number of facilitated ACP discussions with the nurse facilitator, preference for the ACP intervention and baseline eligibility for LTOT. For the same group of participants, factors associated with informal ACP uptake postintervention included participation in two or more discussions with the nurse facilitator, preference for the ACP 
Table 3 Descriptive characteristics of the facilitated ACP intervention

\begin{tabular}{|c|c|c|c|c|}
\hline & \multicolumn{2}{|c|}{$\begin{array}{l}\text { Metropolitan site } \\
\mathrm{N}=48 \text { participants } \\
\mathrm{N}=32 \text { discussions }\end{array}$} & \multicolumn{2}{|c|}{$\begin{array}{l}\text { Rural site } \\
\mathrm{N}=58 \text { participants } \\
\mathrm{N}=109 \text { discussions }\end{array}$} \\
\hline \multicolumn{5}{|l|}{ Timing of first ACP discussion (\% of participants) } \\
\hline $\mathrm{N}(\%)<14$ days postconsent & \multicolumn{2}{|l|}{$22(46 \%)$} & \multicolumn{2}{|l|}{$58(100 \%)$} \\
\hline$N(\%) 14-60$ days postconsent & \multicolumn{2}{|l|}{$7(15 \%)$} & \multicolumn{2}{|l|}{-} \\
\hline $\mathrm{N}(\%)>60$ days postconsent & \multicolumn{2}{|l|}{$2(4 \%)$} & \multicolumn{2}{|l|}{-} \\
\hline \multicolumn{5}{|c|}{ Number of nurse-led ACP discussions (\% of participants) } \\
\hline $\mathrm{N}(\%)$ no discussions & \multicolumn{2}{|l|}{$17(35 \%)$} & \multicolumn{2}{|l|}{-} \\
\hline $\mathrm{N}(\%)$ one discussion & \multicolumn{2}{|l|}{$29(60 \%)$} & \multicolumn{2}{|l|}{$18(31 \%)$} \\
\hline $\mathrm{N}(\%)$ two discussions & \multicolumn{2}{|l|}{$2(4 \%)$} & \multicolumn{2}{|l|}{$29(50 \%)$} \\
\hline $\mathrm{N}(\%)$ three or more discussions & \multicolumn{2}{|l|}{-} & \multicolumn{2}{|l|}{$11(19 \%)$} \\
\hline \multicolumn{5}{|c|}{ People involved in ACP discussion (\% of participants) } \\
\hline $\mathrm{N}(\%)$ with family/carer present once or more & \multicolumn{2}{|l|}{$20(42 \%)$} & \multicolumn{2}{|l|}{$21(36 \%)$} \\
\hline \multicolumn{5}{|l|}{ Duration of ACP discussions (\% of discussions) } \\
\hline $\mathrm{N}(\%)<45 \min$ & \multicolumn{2}{|l|}{$7(22 \%)$} & \multicolumn{2}{|l|}{$87(80 \%)$} \\
\hline $\mathrm{N}(\%)$ 45-90 $\min$ & \multicolumn{2}{|l|}{$22(69 \%)$} & \multicolumn{2}{|l|}{$22(20 \%)$} \\
\hline $\mathrm{N}(\%)>90 \mathrm{~min}$ & \multicolumn{2}{|l|}{$2(6 \%)$} & \multicolumn{2}{|l|}{-} \\
\hline \multicolumn{5}{|l|}{ Location of ACP discussion (\% of discussions) } \\
\hline $\mathrm{N}(\%)$ discussions inpatient setting & \multicolumn{2}{|l|}{$2(6 \%)$} & \multicolumn{2}{|l|}{$8(7 \%)$} \\
\hline $\mathrm{N}(\%)$ discussions outpatient setting & $30(94 \%)$ & & $1(1 \%)$ & \\
\hline $\mathrm{N}(\%)$ discussions general practice clinic & - & & $12(11 \%)$ & \\
\hline $\mathrm{N}(\%)$ discussions home visit & - & & $65(60 \%)$ & \\
\hline N (\%) discussions telephone call & - & & $23(21 \%)$ & \\
\hline ACP domains discussed ( $\%$ of discussions) & Partly & Partly or fully & Partly & Partly or fully \\
\hline $\mathrm{N}(\%)$ understanding current health state & $32(100 \%)$ & $31(97 \%)$ & $109(100 \%)$ & $84(77 \%)$ \\
\hline $\mathrm{N}(\%)$ understanding prognosis & $32(100 \%)$ & 17 (53\%) & $103(95 \%)$ & $56(51 \%)$ \\
\hline $\mathrm{N}(\%)$ perspective on 'living well' & $32(100 \%)$ & $28(88 \%)$ & $109(100 \%)$ & $109(100 \%)$ \\
\hline $\mathrm{N}(\%)$ understanding future treatments & $32(100 \%)$ & $31(97 \%)$ & $105(96 \%)$ & $64(59 \%)$ \\
\hline $\mathrm{N}(\%)$ values and goals of care & $32(100 \%)$ & $29(91 \%)$ & $108(99 \%)$ & 102 (94\%) \\
\hline N (\%) wishes for critical care & $32(100 \%)$ & $32(100 \%)$ & $103(94 \%)$ & $64(59 \%)$ \\
\hline $\mathrm{N}(\%)$ wishes for "trial of treatment" & $30(94 \%)$ & $25(78 \%)$ & $79(72 \%)$ & $44(40 \%)$ \\
\hline $\mathrm{N}(\%)$ cultural, spiritual, religious beliefs & $31(97 \%)$ & $21(66 \%)$ & $56(51 \%)$ & $21(19 \%)$ \\
\hline $\begin{array}{l}\text { Proportions are calculated 'by participant' (number of } p \\
\text { ACP intervention) or 'by discussion' (number of discuss } \\
\text { facilitators). } \\
\text { The discussions undertaken by the nurse ACP facilitat } \\
\text { discussions with loved ones and doctors. } \\
\text { ACP, advance care planning. }\end{array}$ & $\begin{array}{l}\text { ints as a prop } \\
\text { s a proportion } \\
\text { art of the int }\end{array}$ & $\begin{array}{l}\text { of the } 106 \text { partici } \\
141 \mathrm{ACP} \text { discus } \\
\text { on are separate } \mathrm{fr}\end{array}$ & $\begin{array}{l}\text { assigned to rec } \\
\text { conducted by th } \\
\text { e outcome mea }\end{array}$ & $\begin{array}{l}\text { the facilitated } \\
\text { Irse ACP } \\
\text { s of ACP }\end{array}$ \\
\hline
\end{tabular}

intervention, more severe shortness of breath, lower social support and presence of a family member or carer at one or more of the facilitated ACP discussions.

\section{DISCUSSION}

This study assessed the effectiveness of systematic identification of patients with advanced respiratory disease using a tool modified from the Gold Standard Framework to identify those at high risk of death within 12 months, ${ }^{32}$ and proactive intervention through nurse-led ACP discussions. The intervention was effective in increasing formal and informal ACP uptake, particularly among those with a pre-existing preference to receive the intervention.

The inclusion of a preference arm most likely increased participation and retention rates, and more closely reflects real-world situations, in which approaches to care are negotiated between clinicians and patients. In practice, there will always be patients who have strong attitudes about ACP and this trial design enabled recruitment of those who were unwilling to be randomised, potentially improving generalisability. The nature of the ACP intervention made it impossible to blind participants to their allocation, and hence a preference design may have avoided distress among those who wanted a particular type of care but were not offered it. The preference arm introduces a self-selection bias which should be considered when interpreting study findings. ${ }^{44}$

\section{Facilitated ACP intervention}

The ACP intervention differed somewhat across the study sites due to practical factors. In the metropolitan setting, the intervention was delivered in outpatient 
Table 4 Proportion of participants with formal (completion of formal AD or formal nomination of SDM) or informal ACP uptake (discussion with doctor about wishes relating to life-sustaining treatment) at baseline and 6-month follow-up (self-report supplemented by medical notes audit for participants lost to follow-up)

\begin{tabular}{lccc}
\hline & $\begin{array}{l}\text { Baseline } \\
\text { Type of ACP uptake (by condition) }\end{array}$ & $\begin{array}{l}\text { Follow-up } \\
\mathbf{N}(\%)\end{array}$ & p Value \\
\hline Pref-ACP $(\mathrm{N}=61)$ & $1 / 61(2 \%)$ & $42 / 61(69 \%)$ & $<0.001$ \\
$\quad$ Formal ACP uptake & $25 / 61(41 \%)$ & $50 / 61(82 \%)$ & $<0.001$ \\
$\quad$ Informal ACP uptake & $0 / 45(0 \%)$ & $12 / 45(27 \%)$ & $<0.001$ \\
Rand-ACP (N=45) & $8 / 45(26 \%)$ & $26 / 45(58 \%)$ & $<0.001$ \\
$\quad$ Formal ACP uptake & $0 / 22(0 \%)$ & $4 / 22(18 \%)$ & 0.04 \\
$\quad$ Informal ACP uptake & $9 / 22(41 \%)$ & $14 / 22(64 \%)$ & 0.13 \\
Rand-CON (N=22) & & & 0.55 \\
$\quad$ Formal ACP uptake & $1 / 21(5 \%)$ & $2 / 21(10 \%)$ & 0.26 \\
$\quad$ Informal ACP uptake & $3 / 21(14 \%)$ & $6 / 21(29 \%)$ & \\
Pref-CON (N=21) & & \\
$\quad$ Formal ACP uptake & &
\end{tabular}

Figure 2 Self-reported 'readiness' to complete an advance directive among participants assigned to different study groups over time. The sample is limited to participants $(\mathrm{N}=89)$ who responded to baseline, 3-month and 6-month follow-up surveys.

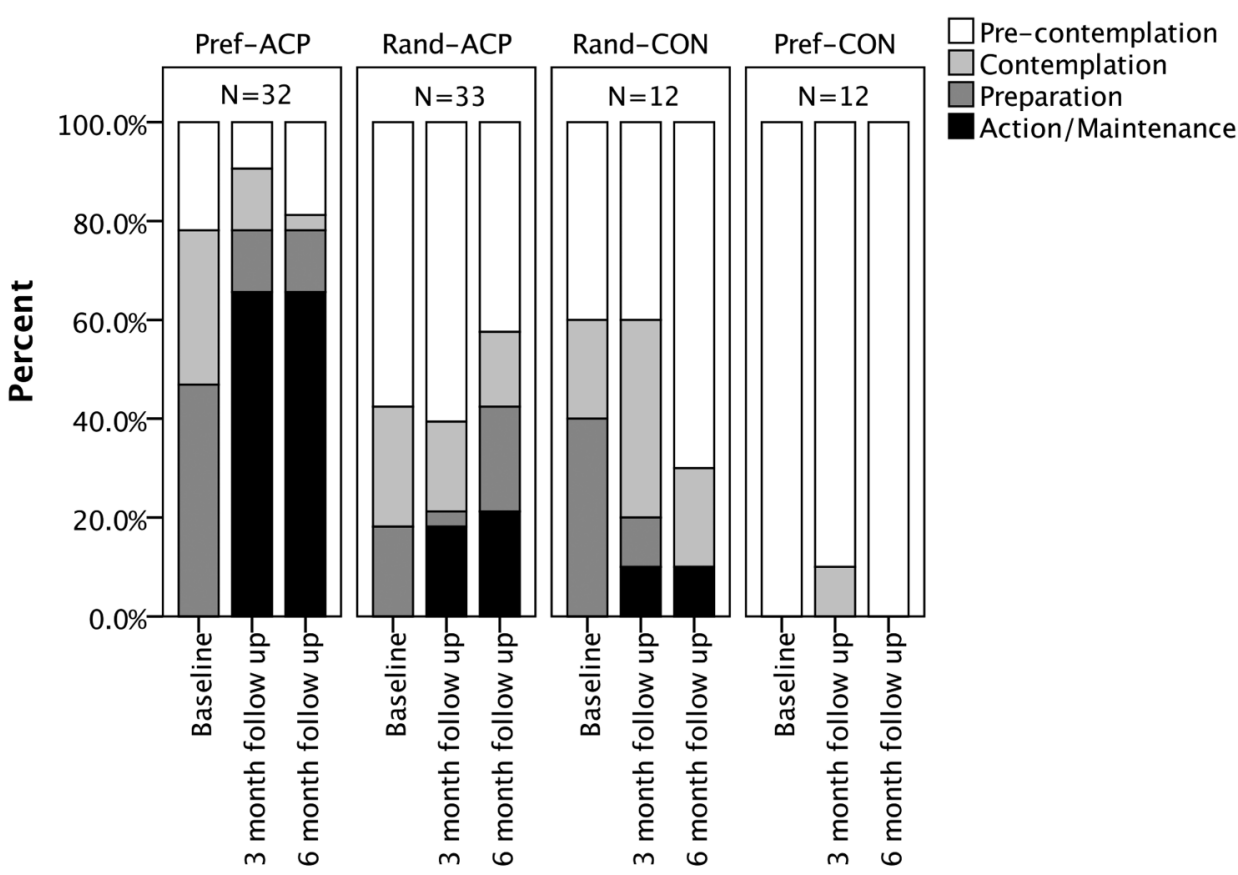

Time

clinics resulting in barriers to participation, particularly for participants with significant symptoms or mobility limitations. In the rural setting, the nurse facilitator was able to visit participants in a range of settings; this resulted in a tendency for multiple ACP discussions, often of shorter duration. Previous research has suggested that community-based ACP delivery can accommodate the needs of patients with heavy symptom burden and enable ongoing ACP discussion. ${ }^{45}$ However, in the current study, a lower than anticipated recruitment rate meant that it was not possible to stratify the data in order to assess the impact of the study setting on the primary outcome.

While the nurses routinely discussed participants' perspectives on 'living well', discussion about prognosis and cultural, spiritual or religious beliefs occurred less often. This may reflect participant preferences for certain domains of discussion, or the nurses' preferences to avoid aspects of discussion that might be seen as the traditional domain of doctors or spiritual advisors. ${ }^{46} 47$ understanding of their illness, values, goals of care and 
Table 5 Final regression models showing factors associated with completion of ACP discussions with loved ones and doctors at baseline, and factors associated with completion of ACP discussion with doctors, or formal ACP documents (formal ACP) at follow-up among those assigned to the intervention

\begin{tabular}{|c|c|c|}
\hline ACP domain & OR & $95 \% \mathrm{Cl}$ \\
\hline \multicolumn{3}{|c|}{ Baseline completion of ACP discussion with loved ones (all participants) } \\
\hline Social support (ENRICHD scale) & 1.10 & 1.04 to 1.65 \\
\hline Education level (completed secondary school) & 1.84 & 0.89 to 3.82 \\
\hline \multicolumn{3}{|l|}{ Baseline completion of ACP discussion with doctors (all participants) } \\
\hline Health related quality of life (EQ-5D-5L index score) & 0.14 & 0.03 to 0.64 \\
\hline COPD diagnosis & 1.94 & 0.86 to 4.36 \\
\hline Gender (female vs male) & 1.83 & 0.87 to 3.85 \\
\hline \multicolumn{3}{|c|}{ Postintervention informal ACP uptake (of those assigned to ACP intervention) } \\
\hline (Baseline) social support (ENRICHD scale) & 0.85 & 0.75 to 0.96 \\
\hline Participation in $\geq 2$ facilitated ACP discussions (vs none) & 5.18 & 1.07 to 25.1 \\
\hline Family/carer involved in a facilitated ACP discussion (vs not) & 4.34 & 1.21 to 15.5 \\
\hline (Baseline) MRC shortness of breath grade 4-5 (vs not) & 4.25 & 1.42 to 12.7 \\
\hline Preferential assignment to ACP intervention (vs random) & 3.32 & 1.18 to 9.33 \\
\hline \multicolumn{3}{|c|}{ Postintervention formal ACP uptake (of those assigned to ACP intervention) } \\
\hline Participation in $\geq 2$ facilitated ACP discussions (vs none) & 7.02 & 1.73 to 35.1 \\
\hline Participation in one facilitated ACP discussion (vs none) & 4.53 & 1.12 to 18.3 \\
\hline Preferential assignment to ACP intervention (vs random) & 5.81 & 2.29 to 14.7 \\
\hline (Baseline) eligibility for LTOT (vs ineligible) & 3.29 & 1.22 to 8.88 \\
\hline
\end{tabular}

\section{ACP uptake and readiness}

The facilitated ACP intervention was associated with higher rates of formal and informal ACP uptake, particularly among those with a strong preference for the intervention, and those who engaged in multiple sessions with the nurse facilitator. Among those randomly allocated to receive the intervention, uptake was not significantly higher than among those assigned to usual care, suggesting that the intervention was particularly effective among those with a preference for ACP. However, it should also be noted that it was rare for patients to be excluded at the screening stage on the basis of prior formal $\mathrm{AD}$ completion (see figure 1). This suggests that a preference for ACP, on its own, does not necessarily lead to high levels of formal ACP uptake, in the absence of a facilitating intervention.

Most participants had discussed ACP with loved ones at baseline, with rates comparable to previous research in a healthy elderly cohort from the USA. ${ }^{27}$ Discussion with loved ones at baseline was associated with social support, ${ }^{29}{ }^{48}$ suggesting that supportive social networks may enable informal discussions outside of clinical relationships. Discussions with loved ones also increased across the follow-up period, regardless of assignment to the intervention. This cohort effect may be associated with disease progression, or may have been prompted by the inclusion of questions about 'ACP readiness' in follow-up surveys.

Consistent with previous research, rates of ACP discussions with doctors were significantly lower than completion of formal ACP documents or discussion with loved ones. ${ }^{27} 30$ The lower rates of discussion with doctors in practice contrast with those studies that suggest that patients prefer to discuss ACP with their trusted doctor. ${ }^{20}{ }^{49}$ However, this paradox does seem to be overcome somewhat by the nurse facilitation process, as the rate of informal ACP uptake (documented discussion with doctor about preferences for life-sustaining treatments) did increase among participants assigned to the intervention, and was associated with having undertaken two or more nurse-facilitated ACP discussions. Similarly, higher rates of ACP discussions with doctors occurred among participants who had a family/carer involved in at least one of the facilitated ACP discussions. This implies that the presence of family/carers in initial ACP discussions prompts further follow-up with the doctor.

On the other hand, participants with lower social support were also more likely to have follow-up ACP discussions with doctors, perhaps as these participants are identified as potentially vulnerable and hence managed more proactively. Previous research has found that patients with more severe symptom burden and lower levels of social support influence GP decisions to initiate ACP discussions. ${ }^{50}$ Social support is typically lower among patients with declining health status, ${ }^{51}$ and health professionals may need to proactively initiate ACP discussion among patients whose illness has led to losses in social support networks. The links between social support and ACP uptake require further investigation.

\section{Limitations}

This study has a number of limitations to consider. Assignment to study arm was unblinded, and the 
preference design introduced a self-selection bias, which complicates interpretation of the data. There is additional complexity in the interpretation of ACP uptake over time due to attrition during the follow-up period (mostly associated with patients dying). This was addressed where possible by supplementing self-report measures with information obtained from follow-up medical notes audits. For measures in which self-report was the only source of data (eg, ACP discussions with loved ones), 3 months was designated as the follow-up point, to minimise the impact of attrition. Where results are reported longitudinally (eg, figure 2), the sample is limited to those who were able to respond at all follow-up time points. Challenges to recruitment meant that the overall study sample was smaller than anticipated and data were collapsed across the two recruitment sites, preventing comparisons across sites. The same nurses who facilitated the ACP intervention also collected follow-up survey responses and undertook medical notes audits; this may be a source of bias. Despite this, high rates of survey (78\% among surviving patients) and notes audit (98\%) completion have yielded detailed data on ACP uptake among a specific cohort of patients with advanced respiratory disease. The authors suggest that the ongoing relationship between the nurse and participant contributed to the high rates of successful follow-up, and may also have facilitated high-quality ACP discussions. ACP interventions that occur outside an ongoing clinical relationship may be less effective, and may require more resources to build patient rapport.

\section{Implications}

This study has a number of implications for practice. A systematic screening process to identify patients with end-stage lung disease was found to be acceptable to clinicians and participants, and was sensitive in detecting patients at high risk of death in the coming year. Many participants expressed a strong preference to receive the ACP intervention, suggesting that assessing preference at the time of screening may enable effective tailoring of ACP interventions. Assignment to the facilitated ACP intervention was associated with large increases in ACP uptake, particularly among those with a pre-existing strong preference for receiving the intervention, and those who engaged in multiple sessions, or involved a family member or carer in the discussion. These findings suggest that facilitated ACP discussions with trained nurses are acceptable to patients with advanced respiratory disease, and effective in promoting ACP uptake. ${ }^{6}$ Programmes aiming to promote ACP uptake may consider assessing patient social support, to identify preferred support people for participation in discussions and to flag patients who may require more intensive support in undertaking ACP.

\section{Author affiliations}

${ }^{1}$ Rural Clinical School of Western Australia, University of Western Australia, Albany, Western Australia, Australia
${ }^{2}$ Rural Clinical School of Western Australia, University of Western Australia, Perth, Western Australia, Australia

${ }^{3}$ Respiratory Department, Sir Charles Gairdner Hospital, Nedlands, Western Australia, Australia

${ }^{4}$ School of Nursing and Midwifery, Edith Cowan University, Perth, Western Australia, Australia

${ }^{5}$ Department of Health, WA Cancer and Palliative Care Network, Perth, Western Australia, Australia

${ }^{6}$ Western Australian Country Health Service, District Health Advisory Committee, Albany, Western Australia, Australia

Twitter Follow Craig Sinclair @csinclair28

Acknowledgements The authors would like to acknowledge the participating patients, carers, clinicians and healthcare facilities.

Contributors CS contributed to study design, grant writing, ethics application and site approvals, contributed to ACP training resource development and adaptation to the local context, conducted data analysis and drafted the manuscript. KAA contributed to study design, grant writing, ethics application and rural site approvals, contributed to ACP training resource development and adaptation, facilitated patient recruitment, provided clinical leadership to guide intervention delivery, contributed to preliminary data analysis, and revised the manuscript. SFE contributed to study design, supervised statistical analysis, revised the manuscript. FW led patient recruitment in rural setting, delivered the ACP intervention, collected data, contributed to preliminary data analysis. SD led patient recruitment in metropolitan setting, delivered the ACP intervention, collected data, contributed to preliminary data analysis. AW contributed to study design, grant writing, contributed to preliminary data analysis, revised the manuscript. KG contributed to study design, ACP training resource development and adaptation to local setting, contributed to preliminary data analysis, revised the manuscript. AK contributed to study design, grant writing, ethics application and site approvals, contributed to ACP training resource development and adaptation to local setting, contributed to preliminary data analysis. DP contributed to study design, ethics application and site approvals, contributed to ACP training resource development and adaptation to local setting, commented on draft manuscript from a consumer perspective. FB contributed to study design, grant writing, ethics application and metropolitan site approvals, contributed to ACP training resource development and adaptation, facilitated patient recruitment, provided clinical leadership to guide intervention delivery, contributed to preliminary data analysis, revised the manuscript. All authors reviewed the final manuscript and have approved it for submission.

Funding This work was supported by a grant from the WA Health Department State Health Research Advisory Committee RA/1/467/104.

\section{Competing interests None declared.}

Ethics approval The study was approved by the Human Research Ethics Committees (HREC) at Sir Charles Gairdner Hospital (2013/173), University of WA (4/1/6547), WA Country Health Service (WACHS 1305) and the WA Department of Health (2014/46).

Provenance and peer review Not commissioned; externally peer reviewed.

Data sharing statement Owing to a planned linkage of these data with healthcare usage data held by the WA Department of Health, there are currently restrictions on the open publication of the raw data reported here.

Open Access This is an Open Access article distributed in accordance with the Creative Commons Attribution Non Commercial (CC BY-NC 4.0) license, which permits others to distribute, remix, adapt, build upon this work noncommercially, and license their derivative works on different terms, provided the original work is properly cited and the use is non-commercial. See: http:// creativecommons.org/licenses/by-nc/4.0/

\section{REFERENCES}

1. Sudore RL, Fried TR. Redefining the "planning" in advance care planning: preparing for end-of-life decision making. Ann Intern Med 2010;153:256-61. 
2. Sudore RL, Stewart AL, Knight SJ, et al. Development and validation of a questionnaire to detect behavior change in multiple advance care planning behaviors. PLOS ONE 2013;8:e72465.

3. Bischoff KE, Sudore R, Miao $\mathrm{YH}$, et al. Advance care planning and the quality of end-of-life care in older adults. J Am Geriatr Soc 2013;61:209-14.

4. Brinkman-Stoppelenburg A, Rietjens JA, van der Heide A. The effects of advance care planning on end-of-life care: a systematic review. Palliat Med 2014;28:1000-25.

5. Houben CHM, Spruit MA, Groenen MTJ, et al. Efficacy of advance care planning: a systematic review and meta-analysis. J Am Med Dir Assoc 2014;15:477-89.

6. Detering KM, Hancock AD, Reade MC, et al. The impact of advance care planning on end of life care in elderly patients: randomised controlled trial. BMJ 2010;340:c1345.

7. Wong JS-h, Gottwald M. Advance care planning discussions in chronic obstructive pulmonary disease: a critical review. J Palliat Care 2015;31:258-64.

8. Nguyen M, Chamber-Evans J, Joubert A, et al. Exploring the advance care planning needs of moderately to severely ill people with COPD. Int J Palliat Nurs 2013;19:389-95.

9. Janssen DJA, Engelberg RA, Wouters EFM, et al. Advance care planning for patients with COPD: past, present and future. Patient Educ Couns 2012;86:19-24.

10. Habraken JM, ter Riet G, Gore JM, et al. Health-related quality of life in end-stage COPD and lung cancer patients. J Pain Symptom Manag 2009;37:973-81.

11. Weingaertner V, Scheve $C$, Gerdes V, et al. Breathlessness, functional status, distress, and palliative care needs over time in patients with advanced chronic obstructive pulmonary disease or lung cancer: a cohort study. J Pain Symptom Manag 2014;48:569-81.e1.

12. Nur U, Quaresma M, De Stavola B, et al. Inequalities in non-small cell lung cancer treatment and mortality. $J$ Epidemiol Community Health 2015;69:985-92.

13. Groenewegen $\mathrm{KH}$, Schols AMWJ, Wouters EFM. Mortality and mortality-related factors after hospitalization for acute exacerbation of COPD. Chest 2003;124:459.

14. Claessens MT, Lynn J, Zhong Z, et al. Dying with lung cancer or chronic obstructive pulmonary disease: Insights from SUPPORT. Study to understand prognoses and preferences for outcomes and risks of treatments. J Am Geriatr Soc 2000;48:S146-S53.

15. Janssen DJA, Curtis JR, Au DH, et al. Patient-clinician communication about end-of-life care for Dutch and US patients with COPD. Eur Respir J 2011;38:268-76.

16. Pardon K, Deschepper R, Vander Stichele R, et al. Preferred and actual involvement of advanced lung cancer patients and their families in end-of-life decision making: a multicenter study in 13 hospitals in Flanders, Belgium. J Pain Symptom Manag 2012:43:515-26.

17. Gardiner C, Gott M, Payne S, et al. Exploring the care needs of patients with advanced COPD: an overview of the literature. Respir Med 2010:104:159-65.

18. Crawford GB, Brooksbank MA, Brown M, et al. Unmet needs of people with end-stage chronic obstructive pulmonary disease: recommendations for change in Australia. Intern Med $J$ 2013;43:183-90.

19. Patel K, Janssen DJA, Curtis JR. Advance care planning in COPD. Respirology 2012;17:72-8.

20. Pfirstinger J, Kattner D, Edinger M, et al. The Impact of a tumor diagnosis on patients' attitudes toward advance directives. Oncology 2014;87:246-56

21. Knauft E, Nielsen EL, Engelberg RA, et al. Barriers and facilitators to end-of-life care communication for patients with COPD. Chest 2005;127:2188-96.

22. Rhee JJ, Zwar NA, Kemp LA. Advance care planning and interpersonal relationships: a two-way street. Fam $\mathrm{Pr}$ 2013;30:219-26.

23. Gott M, Gardiner C, Small N, et al. Barriers to advance care planning in chronic obstructive pulmonary disease. Palliat Med 2009;23:642-8.

24. Fletcher S, Sinclair C, Rhee J, et al. Rural health professionals' experiences in implementing advance care planning: a focus group study. Aust J Prim Health 2015;22:423-7.

25. Fried TR, Bullock K, lannone L, et al. Understanding advance care planning as a process of health behavior change. J Am Geriatr Soc 2009:57:1547-55.
26. Sudore RL, Schickedanz AD, Landefeld CS, et al. Engagement in multiple steps of the advance care planning process: a descriptive study of diverse older adults. J Am Geriatr Soc 2008;56:1006-13.

27. Fried TR, Redding CA, Robbins ML, et al. Stages of change for the component behaviors of advance care planning. J Am Geriatr Soc 2010;58:2329-36.

28. Fried TR, Redding CA, Robbins ML, et al. Promoting advance care planning as health behavior change: development of scales to assess decisional balance, medical and religious beliefs, and processes of change. Patient Educ Couns 2012;86:25-32.

29. Sinclair C, Auret KA, Burgess A. The balancing point: understanding uptake of advance directive forms in a rural Australian community. BMJ Support Palliat Care 2013;3:358-65.

30. Amjad H, Towle V, Fried T. Association of experience with illness and end-of-life care with advance care planning in older adults. J Am Geriatr Soc 2014;62:1304-9.

31. Brewin CR, Bradley C. Patient preferences and randomized clinical-trials. BMJ 1989;299:313-15.

32. The Gold Standards Framework Centre in End of Life Care CIC. The GSF Prognostic Indicator Guidance (4th edn). 2011 (14 July 2014) http://www.goldstandardsframework.org.uk/cd-content/uploads/files/ General Files/Prognostic Indicator Guidance October 2011.pdf

33. Oken MM, Creech RH, Tormey DC, et al. Toxicity and response criteria of the Eastern Cooperative Oncology Group. Am J Clin Oncol 1982;5:649-55.

34. Murray SA, Boyd K. Using the 'surprise question' can identify people with advanced heart failure and COPD who would benefit from a palliative care approach. Palliat Med 2011;25:382.

35. Stenton C. The MRC breathlessness scale. Occup Med (Lond) 2008;58:226-7.

36. Auret K, Sinclair C, Averill B, et al. Advance care planning and end-of-life care in a network of rural Australian hospitals. Aust $J$ Rural Health 2015;23:195-200.

37. Department of Health WA. Preparing an advance health directive. East Perth: Department of Health (WA), 2010.

38. Department of Health WA. My Advance Care Plan. East Perth: Department of Health; 2013 [31 March 2016]. http://www.health.wa. gov.au/advancecareplanning/docs/ACP_form.pdf

39. Office of the Public Advocate WA. A guide to enduring power of guardianship in Western Australia. East Perth: Office of the Public Advocate (WA), 2010.

40. van Hout $B$, Janssen MF, Feng $Y S$, et al. Interim scoring for the EQ-5D-5L: mapping the EQ-5D-5L to EQ-5D-3L value sets. Value Health 2012;15:708-15.

41. Guyatt GH, Mitchell A, Molloy DW, et al. Measuring patient and relative satisfaction with level or aggressiveness of care and involvement in care decisions in the context of life-threatening illness. J Clin Epidemiol 1995;48:1215-24.

42. Vaglio J Jr, Conard M, Poston WS, et al. Testing the performance of the ENRICHD Social Support Instrument in cardiac patients. Health Qual Life Outcomes 2004;2:24.

43. Green MJ, Schubart JR, Whitehead MM, et al. Advance care planning does not adversely affect hope or anxiety among patients with advanced cancer. J Pain Symptom Manag 2015;49:1088-96.

44. Torgerson DJ, Sibbald B. Understanding controlled trials-what is a patient preference trial? BMJ 1998;316:360.

45. Ratner E, Norlander L, McSteen K. Death at home following a targeted advance-care planning process at home: the kitchen table discussion. J Am Geriatr Soc 2001;49:778-81.

46. Keall R, Clayton JM, Butow P. How do Australian palliative care nurses address existential and spiritual concerns? Facilitators, barriers and strategies. J Clin Nurs 2014;23:3197-205.

47. Hjelmfors $L$, van der Wal MHL, Friedrichsen MJ, et al. Patient-nurse communication about prognosis and end-of-life care. J Palliat Med 2015;18:865-71.

48. Carr D. "I don't want to die like that...": the impact of significant others' death quality on advance care planning. Gerontologist 2012;52:770-81. doi:10.1093/geront/gns051

49. Ahalt C, Walter LC, Yourman L, et al. "Knowing is better": preferences of diverse older adults for discussing prognosis. J Gen Intern Med 2012;27:568-75.

50. Sinclair C, Gates K, Auret K, et al. Factors influencing Australian general practitioners' clinical decisions regarding advance care planning: a factorial survey. J Pain Symptom Manag 2016;51:718-27.

51. Hyduk CA. The dynamic relationship between social support and health in older adults: assessment implications. J Gerontol Soc Work 1996;27:149-65. 